\title{
Stuhl spenden - Leben retten?
}

- Das Spenden von Organen oder Geweben ist ein heikles Thema. Zwar sind alle dafür, aber wenn es ernst wird, sprich ein Spenderausweis ausgefüllt werden soll, befällt die meisten doch ein mulmiges Gefühl, um nicht zu sagen die Angst, vielleicht lebendig für tot erklärt zu werden. Für alle, die mit einer Spende aus ihrem Körper aber schon zu Lebzeiten etwas Gutes bewirken möchten, bietet sich jetzt eine neue Chance. Die Rede ist von der Fäzesspende, auf gut Deutsch "Stuhlspende“.

Sollte ich mit diesem Thema bei Ihnen ästhetisches Missempfinden provozieren, so tut mir dies natürlich sehr leid. Aber Medizin ist eben nicht immer ein ästhetisches Erlebnis. In der Tat handelt es sich - ob Sie es glauben oder nicht - um eine neue wissenschaftlich untersuchte Behandlungsoption. Vorgestellt und ausführlich kommentiert wurde dieses Thema auch in GastroNews 2010;3:19. Hier wurde über Studien berichtet, bei denen Patienten mit rezidivierender Clostridium-difficile-Infektion mit der Fäzes von gesunden Spendern behandelt wurden. Die Autoren schlussfolgern, die Transplantation von Fäzes bei solchen Patienten stelle neben der Immunisierung einen vielversprechenden Therapieansatz dar, auch wenn noch kein abschließendes Votum gegeben werden könne.

\section{Mehr Spender als Empfänger}

Noch ein paar Worte zur Methodik: Der gewonnene Stuhl wurde mit Wasser oder Kochsalzlösung verdünnt und dann entweder über ein Klysma bzw. eine Koloskopie und in ca. 20\% über eine nasogastrale (!), duodenale oder jejunale Sonde in den erkrankten Darm transferiert. Leider enthält die Studie keine Aussagen darüber, ob die Patienten ausführlich über diese Therapie aufgeklärt wurden und welche Akzeptanz das Verfahren gefunden hat. Hand aufs Herz: Würden Sie sich so behandeln lassen, auch wenn die Sache die höchste Evidenzstufe erreichen würde? Ich wage die Prognose, dass bei dieser „Transplantation“ erstmals die Zahl der Spender deutlich höher liegen wird als die der Empfänger. Wartelisten sind also kaum zu erwarten.

Dr. med. Peter Stiefelhagen .

ALKOHOL IN DER SCHWANGERSCHAFT

\section{Je älter und gebildeter, desto geringer die Einsicht}

- Mehr als $80 \%$ der Frauen im gebärfähigen Alter nehmen mehr oder weniger regelmäßig alkoholische Getränke zu sich, und trotz aller Aufklärungsbemühungen sind nur $42 \%$ aller werdenden Mütter abstinent. Überraschenderweise steigt der Alko-

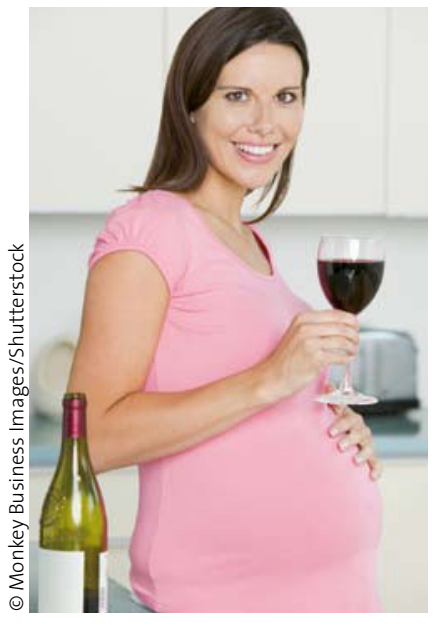

Zum Wohl - aber nicht für das Kind. holkonsum in der Schwangerschaft signifikant mit Alter und Schulabschluss der Frauen, wie eine Untersuchung in Berlin ergab. Schwangere über 35 Jahren mit Abitur konsumierten am häufigsten alkoholische Getränke.

Etwa 3000 Kinder kommen in Deutschland jährlich mit schweren Entwicklungsstörungen aufgrund von Alkoholkonsum der Mutter zur Welt. Da „bisher nicht bekannt ist, wann in der Schwangerschaft welche Menge Alkohol gefährlich ist“, rät Prof. Dr. Klaus Friese, München, 1. Vizepräsident der Deutschen Gesellschaft für Gynäkologie und Geburtshilfe (DGGG), Schwangeren zu völliger Abstinenz. Mo - Pressemitteilung der DGGG

EINE FRAGE DER OBERFLÄCHE

\section{Warum Dicke so schwer abnehmen}

- Warum können manche Menschen essen wie ein Scheunendrescher und trotzdem schlank bleiben, während andere ein Stück Torte scheinbar nur ansehen müssen, um zuzunehmen? Wissenschaftler der Uni Gießen haben das Phänomen untersucht, weshalb es dicken Menschen so schwerfällt abzunehmen und warum schlanke Menschen andererseits nur schwer zunehmen. Beide Effekte sind physikalisch erklärbar, meinen die Autoren um Prof. Elmar Schlich. Mithilfe von 3-D-Body-Scan-Messungen hatten sie die spezifische Körperoberfläche, d.h. das Verhältnis der tatsächlichen Körperoberfläche zur Körpermasse, von 188 Probanden untersucht. Da die spezifische Körperoberfläche bei Dicken um 50\% kleiner ist als bei Dünnen, geben Übergewichtige pro Kilogramm sehr viel weniger Wärme an die Umgebung ab als die Schlanken - und bleiben eher dick. Schlanke wiederum strahlen wegen ihrer relativ großen Oberfläche sehr viel Wärme ab, brauchen entsprechend mehr Energie, um ihre Körpertemperatur aufrechtzuerhalten - und behalten daher auch trotz gelegentlicher kulinarischer Sünden ihre schlanke Linie.

MO = 